欧

Experiencia Clínica

\title{
Endocarditis Infecciosa: caracterización clínica de la enfermedad. Revisión de casos de los últimos 5 años
}

\author{
Pablo Flores ${ }^{1}$, Nicolás González ${ }^{2}$, Pablo Betancourt ${ }^{1,3}$, Javiera Berho ${ }^{4}$, Carlos Astudillo ${ }^{5}$, Cecilia Garcia ${ }^{5}$ y Juan Rojas ${ }^{5}$. \\ 1. Médico internista, Unidad de paciente crítico del Hospital Carlos Van Buren de Valparaíso. \\ 2. Residente de Medicina interna de la Universidad de Valparaíso, Servicio de Medicina interna del Hospital Carlos Van Buren de Valparaíso. \\ 3. Profesor ayudante de la cátedra de Salud Pública, Universidad de Valparaíso. \\ 4. Residente de Inmunología de la Universidad de Chile, Servicio de Medicina interna del Hospital Clínico Universidad de Chile. \\ 5. Cardiólogo, Servicio de Cardiología del Hospital Carlos Van Buren de Valparaíso.
}

${ }^{*}$ Declaramos no tener conflictos de interés.

Antecedentes: La Endocarditis infecciosa es una enfermedad grave y su incidencia ha aumentado en el último tiempo. En Chile faltan estudios observacionales que representen adecuadamente nuestra realidad local y nacional.

Objetivo: Describir las características clínicas, ecográficas y bacteriológicas de los pacientes con Endocarditis Infecciosa del Hospital Carlos Van Buren de Valparaíso, entre los años 2012 y 2016.

Métodos: Se realizó un análisis descriptivo de 35 pacientes con diagnóstico de Endocarditis infecciosa, recolectándose variables clínicas, imagenológicas y de laboratorio. Para describir los resultados se utilizó frecuencias, medianas y figuras.

Resultados: Se observó un aumento de casos de
Endocarditis infecciosa en los últimos dos años. Del total de pacientes con Endocarditis infecciosa, 28 casos $(80 \%)$ eran de válvula nativa, siendo el compromiso más frecuente el de válvula aórtica. Se observó un aumento de las comorbilidades no cardíacas y de los casos provocados por el grupo Staphylococcus. Los síntomas más comunes fueron compromiso del estado general y fiebre, y las complicaciones más frecuentes fueron insuficiencia renal aguda, insuficiencia cardíaca aguda y fenómenos embólicos. Un total de 14 pacientes fallecieron producto de la infección, las complicaciones y cirugía. Conclusiones: Se realiza una caracterización actualizada de la enfermedad. Se necesitan más estudios con un mayor número de paciente para un mejor entendimiento de nuestra realidad.

\section{Correspondencia:}

Dr. Pablo Flores Fernández

Dirección: San Ignacio № 725, Hospital Carlos Van Buren, Valparaíso

Cel.: 56-322364000, 56-942889594

pablo.flofer@gmail.com 


\section{Infective endocarditis: clinical experience in the last five years}

Background: Infective endocarditis is a severe illness the incidence of which has increased over time. There are relatively few observational studies that adequately represent the characteristics of the disease in Chile.

Aim: To describe clinical, echographic and bacteriological characteristics of patients with infective endocarditis at Carlos van Buren Hospital in Valparaíso, between 2012 and 2016.

Methods: A descriptive analysis of 35 patients diagnosed with infective endocarditis was performed. Cinical, imaging findings and laboratory variables were collected. Relative frequencies, medians and figures were used to describe the results.

Results: We observed an increase in cases of infective endocarditis in the last two years. Of all patients diagnosed with infective endocarditis, $80 \%$ had native valve involvement, more frequently at the aortic valve. There was an increase in non-cardiac comorbidities and Staphylococcus was identified as the main etiology. Weakness and fever were the most common symptoms, whereas more common complications were acute kidney and heart failure and embolic phenomena. A total of 14 patients died of infection, complications or after surgery surgery.

Conclusions: An updated characterization of Infective endocarditis at a Chilean hospital is presented. However, more studies are needed with a larger number of patients for a better characterization of the disease in our country.

Key words: Infective endocarditis; heart valves. 


\section{Introducción:}

La endocarditis infecciosa (EI) es una enfermedad grave que afecta el endocardio y es potencialmente mortal ${ }^{1}$. En Estados unidos la incidencia varía entre 1.7 - 6.2 casos por 100.000 habitantes al año ${ }^{2,3}$, y en Chile la incidencia es de 2-3 casos por 100.000 habitantes al año, siendo una afección con elevada morbilidad y mortalidad, que asciende a $29 \%$ en series nacionales ${ }^{4}$.

En los últimos años se han producido una serie de cambios epidemiológicos y tecnológicos en el grupo de pacientes susceptibles a EI y en las herramientas médicas disponibles que han determinado que se modifiquen la etiología, momento del diagnóstico, tratamiento, evolución y pronóstico de enfermos con EI. Dentro de éstos cambios se encuentran la disminución de la incidencia de enfermedad reumática ${ }^{5,6}$, el aumento de la edad promedio de presentación ${ }^{7}$, un mayor acceso a procedimientos intervencionistas y mayor utilización de dispositivos como prótesis cardíacas, marcapasos y catéteres intravasculares, aumento de comorbilidades no cardíacas, mayor utilización de drogas ilícitas, mejora en los métodos diagnósticos y precocidad del tratamiento $8,9,10,11$.

En nuestro hospital hemos observado un incremento significativo de los pacientes con EI en los últimos años, superando con creces los previos, lo que ha motivado a efectuar una revisión clínica de nuestros casos, realizar una caracterización clínica de la enfermedad, de tal forma de aportar nuestra experiencia clínica actualizada al medio científico nacional.

\section{Métodos}

Se realizó un análisis descriptivo de una serie de 35 pacientes con diagnóstico de Endocarditis infecciosa del Hospital Carlos Van Buren de Valparaíso entre los años 2012 y 2016. El diagnóstico se basó en los criterios de Duke modificados, en todos los casos ${ }^{12}$. Todos estos casos fueron estudiados con hemocultivos seriados, ecocardiografía e imágenes complementarias como parte del estudio de embolia. Se recolectaron variables clínicas, ecográficas, bacteriológicas y de laboratorio en todos los casos. Las variables fueron: edad, sexo, ritmo, fracción de eyección, válvula afectada, tipo de válvula, y grado de regurgitación. También se consignaron comorbilidades, antecedente de abuso de cocaína, EI previa, procedimiento dental previo, presencia de dispositivos intracardíacos, presencia de fístula arteriovenosa y catéter de hemodiálisis. En los casos operados se tipificó la válvula utilizada, la condición del paciente al egreso, Para describir los resultados se utilizó frecuencias y medianas con su respec- tivo rango intercuartílico (RIC). El análisis estadístico se realizó con el software Stata v12.

\section{Resultados:}

Se evaluaron 35 pacientes con diagnóstico de EI, 19 hombres y 16 mujeres con una mediana de edad de 57 años (RIC 40-70). La distribución de casos por año fue la siguiente: Año 2012 con 2 casos, Año 2013 con 3 casos, Año 2014 con 7 casos, Año 2015 con 13 casos y Año 2016 con 10 casos (Figura 1). 34 pacientes (97\%) tenían ritmo sinusal de base y la mediana de la fracción de eyección fue 0.56 (RIC 0.52-0.7). Todos los pacientes fueron diagnosticados durante la primera semana de hospitalización, pero no se pudo determinar el tiempo desde el inicio de la sintomatología y la consulta. Se utilizó ecocardiografía transtorácica para realizar el diagnóstico en 12 casos (34.29\%) y transesofágica en 23 casos $(65.71 \%)$. En 3 casos $(8.57 \%)$ se encontró el antecedente de EI previa. En 28 casos (80\%) la válvula comprometida era nativa y en 7 casos (20\%) era protésica. El detalle completo de la afectación valvular se muestra en la Tabla 1.

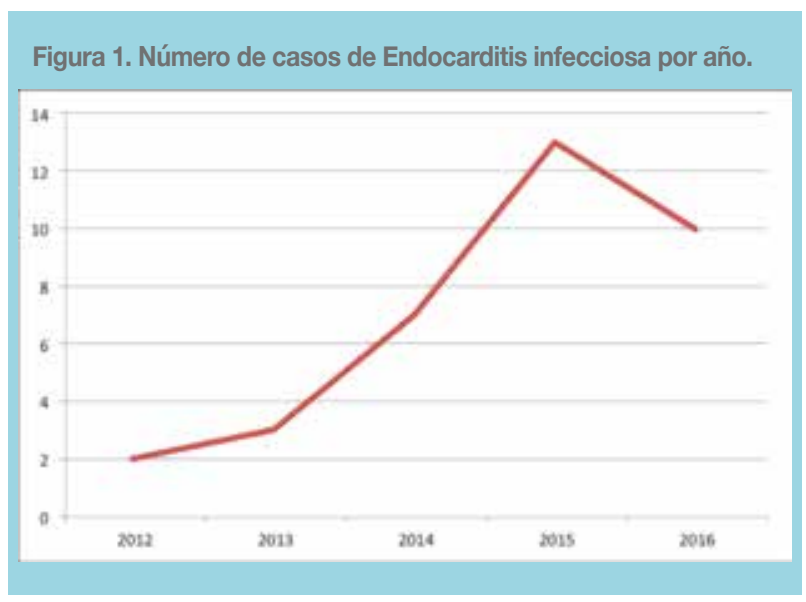

La presentación clínica fue subaguda en 11 casos y aguda en 24 casos. Las principales comorbilidades asociadas se resumen en la Tabla 1. El antecedente de abuso de sustancias (Cocaína) fue positivo en 6 casos (17.1\%). En cuanto a dispositivos intracardíacos, sólo un paciente era portador de marcapasos definitivo. En 5 pacientes $(14.3 \%)$ se observó presencia de fístula arteriovenosa y 3 pacientes $(8.6 \%)$ tenían catéter de hemodiálisis. 11 pacientes (31.4\%) requirieron hospitalización en unidad de paciente crítico. Todos los pacientes recibieron tratamiento antimicrobiano indicado por el equipo de infectología de nuestro hospital y en 10 casos (28.6\%) se requirió cirugía de recambio valvular, de los cuales 7 
Tabla 1. Características generales de los pacientes.

\begin{tabular}{|c|c|c|}
\hline & Mediana (RIC) & Frecuencia (\%) \\
\hline Edad (años) & $57(40-70)$ & \\
\hline Sexo masculino & & $19(54.3)$ \\
\hline Ritmo sinusal & & $34(97.1)$ \\
\hline Fracción de eyección & $0.56(0.52-0.7)$ & \\
\hline Presentación & & \\
\hline Subaguda & & $11(31.4)$ \\
\hline Aguda & & $24(68.657)$ \\
\hline Número de válvulas afectadas & & \\
\hline Monovalvular & & $27(77.1)$ \\
\hline Multivalvular & & $8(22.9)$ \\
\hline Válvula afectada & & \\
\hline Nativa & & \\
\hline Aórtica & & 19 (54.3) \\
\hline Mitral & & $11(31.4)$ \\
\hline Tricúspide & & $3(8.6)$ \\
\hline Protésica & & \\
\hline Mitral (Mecánica) & & $5(14.3)$ \\
\hline Aórtica (Mecánica) & & $4(11.4)$ \\
\hline Pulmonar (Biológica) & & $1(2.9)$ \\
\hline Grado de regurgitación valvular & & \\
\hline Leve & & $9(25.7)$ \\
\hline Moderada & & $10(28.6)$ \\
\hline Severa & & $15(42.9)$ \\
\hline Comorbilidades asociadas: & & \\
\hline Hipertensión arterial & & $15(42.9)$ \\
\hline Cardiopatía hipertensiva & & $12(24.3)$ \\
\hline Diabetes mellitus tipo 2 & & $8(22.9)$ \\
\hline Enf. Valvular degenerativa & & $8(22.9)$ \\
\hline Insuficiencia renal crónica & & $6(17.1)$ \\
\hline Enfermedad reumática & & $5(14.3)$ \\
\hline Enfermedad mental & & $3(8.6)$ \\
\hline Válvula aórtica bicúspide & & $2(5.7)$ \\
\hline Mesenquimopatías & & $2(5.7)$ \\
\hline Tetralogía de Fallot & & $1(2.9)$ \\
\hline Trasplante renal & & $1(2.9)$ \\
\hline Antecedente de abuso de cocaína & & $6(17.1)$ \\
\hline Portador de fístula arteriovenosa & & $5(14.23)$ \\
\hline Portador de catéter de hemodiálisis & & $3(8.6)$ \\
\hline Endocarditis infecciosa previa & & $3(8.6)$ \\
\hline Usuario de marcapasos definitivo & & $1(2.9)$ \\
\hline Procedimiento dental previo & & $1(2.9)$ \\
\hline
\end{tabular}

RIC :Rango intercuatílico.

fueron por insuficiencia cardíaca aguda sin respuesta a tratamiento médico y 3 casos por embolización sistémica recurrente. Del total de pacientes fallecidos (14 casos), 12 de ellos fallecieron producto de complicaciones cardiacas e infecciosas y 2 pacientes producto de la cirugía. Respecto a la microbiología de pacientes fallecidos, en 6 casos se aisló Staphycococcus aureus, 6 casos no fueron identificados, 1 caso con Enterococo faecium y 1 caso con Escherichia coli. Las manifestaciones clínicas de los enfermos se muestran en la Tabla 2, las complicaciones valvulares y sistémicas en la Tabla 3 y la microbiología aislada en la Tabla 4.

\section{Tabla 2. Manifestaciones clínicas de la enfermedad.}

\begin{tabular}{|l|c|}
\hline Compromiso del estado general & Frecuencia (\%) \\
\hline Fiebre & $27(79.4)$ \\
\hline Soplo cardíaco & $25(71.4)$ \\
Dolor abdominal & $23(65.7)$ \\
Fenómenos inmunológicos & $15(42.9)$ \\
Artralgias & $14(40.0)$ \\
Dolor lumbar & $4(11.4)$ \\
Nódulos de Osler & $1(2.9)$ \\
\end{tabular}

Tabla 3. Complicaciones sistémicas y valvulares.

\begin{tabular}{|l|c|}
\hline & Frecuencia (\%) \\
\hline Insuficiencia renal aguda & $19(54.3)$ \\
\hline Insuficiencia cardíaca aguda & $16(45.7)$ \\
Embolia cerebral & $14(40.0)$ \\
Embolia renal & $8(22.9)$ \\
Embolia esplénica & $8(22.9)$ \\
Embolia pulmonar & $1(2.9)$ \\
Shock séptico & $8(23.5)$ \\
Perforación valvular & $2(5.7)$ \\
Absceso valvular & $1(2.9)$ \\
Fístula valvular & $1(2.9)$ \\
\end{tabular}

Tabla 4. Microbiología aislada en hemocultivos.

\begin{tabular}{|l|c|}
\hline Staphylococcus aureus MS & Frecuencia (\%) \\
\hline Streptococcus viridans & $9(25.7)$ \\
\hline Staplycococcus coagulasa negativo & $3(8.6)$ \\
Enterococcus faecium & $2(5.7)$ \\
Enterococcus faecalis & $2(5.7)$ \\
Candida parapsilosis & $2(5.7)$ \\
Streptococcus pneumoniae & $2(5.7)$ \\
Escherichia coli & $1(2.9)$ \\
No identificado & $1(2.9)$ \\
\hline
\end{tabular}

MS: Meticilino sensible. 


\section{Discusión:}

En nuestro estudio observamos un incremento de los casos de EI respecto a los años anteriores, esto es compatible con otras series nacionales e internacionales que ya mostraban esta tendencia ${ }^{4,5}$. Creemos que este incremento se ve explicado, por una parte, por la mayor cantidad de pacientes con comorbilidades no cardíacas que producen inmunosupresión secundaria, como son la diabetes mellitus, insuficiencia renal crónica y mesenquimopatías, pero también porque estamos en un momento histórico en el que el intervencionismo terapéutico está ganando terreno en todas las áreas y subespecialidades médicas, siendo cada vez más frecuente encontrar pacientes con prótesis, catéteres y otros dispositivos corporales, que implican mayores cuidados sanitarios y potenciales puertas de entrada para infecciones.

En nuestra serie encontramos una frecuencia baja de endocarditis infecciosa previa, cardiopatía congénita y enfermedad reumática que según diversas fuentes representan factores de riesgo importantes para desarrollar la infección ${ }^{13}, 14,15$. Estos hallazgos pueden ser explicados por el número bajo de pacientes en nuestro estudio y porque la incidencia de enfermedad reumática ha disminuido con el tiempo ${ }^{2}$. Al contrario, encontramos una frecuencia similar de prótesis valvular con otros estudios nacionales 16,20 . No observamos casos relacionados con el uso de drogas inyectables, siendo esto explicado por el limitado acceso a este tipo de sustancias en nuestro medio. Sin embargo, es destacable que surge de manera frecuente el antecedente de abuso de cocaína y pasta base en pacientes con EI, y por tanto creemos que debería tenerse presente y considerarse como un factor de riesgo importante en nuestro país.

Al igual que otros estudios internacionales, en nuestro reporte los grupos Streptococcus y Staphylococcus dan cuenta de la mayoría de los $\operatorname{casos}^{5}$. Hemos observado un cambio en la bacteriología implicada en nuestro hospital, asemejándonos más a los países desarrollados, en donde las infecciones por el grupo Staphylococcus están superando al grupo Streptococcus. Esto último también se ha observado en varias series extranjeras ${ }^{17,18}$, 19 y en algunas nacionales ${ }^{4}$. Otros estudios nacionales aún muestran más casos debidos al grupo Streptococ$\operatorname{cus}^{20,21}$

Cabe destacar que del total de pacientes fallecidos (14 casos), en 6 casos se aisló Staphylococcus aureus y en otros 6 casos no se pudo identificar un microorganismo, lo que da cuenta de la agresividad del primero y de la importancia pronóstica de tener un microorganismo identificado para instaurar un tratamiento adecuado.

El alto porcentaje de microorganismos no identificados $(37.14 \%)$ es similar al valor reportado por estudios previos nacionales ${ }^{4}$, siendo su posible explicación el uso previo de antibióticos, los problemas técnicos de los medios de cultivo y la ausencia de técnicas moleculares de estudio en nuestro hospital.

Actualmente, la EI de válvula nativa sigue siendo más frecuente que la EI de válvula protésica4. Esta última se observa hasta en el $25 \%$ de los casos en los países desarrollados $^{16}$, y en series nacionales hasta en el $28 \%{ }^{20}$. En nuestro estudio encontramos este antecedente en 7 casos (20\%). La válvula que mayormente se ven afectadas son la válvula aórtica y mitral, tanto para válvula nativa como protésica, siendo este hallazgo similar a los reportados en otras series 20,21 .

En cuanto a las manifestaciones clínicas, los síntomas más frecuentes encontrados en nuestro estudio, como fiebre y compromiso del estado general son bastante inespecíficos, siendo la presentación clásica de la enfermedad infrecuente. Es por esto que se requiere un alto índice de sospecha por parte del clínico y apoyado por estudios imagenológicos y bacteriológicos. En nuestro estudio no se pudo obtener el tiempo transcurrido desde el inicio de los síntomas y la consulta médica.

Dentro de las complicaciones asociadas, la insuficiencia renal, insuficiencia cardíaca y fenómenos embólicos fueron las más frecuentes al igual que en otros estudios 16,20, 21 , pero a diferencia de éstos, en nuestro reporte encontramos una muy baja frecuencia de embolia pulmonar.

Respecto a los métodos diagnósticos imagenológicos, queremos enfatizar el importante rol de la ecocardiografía 3D, que se han posicionado como una gran herramienta diagnóstica al lado de la cama del enfermo, y que ha permitido mejorar el diagnóstico temprano de la infección y facilitar la detección de complicaciones.

A pesar de estos avances tecnológicos, la EI es una infección grave, con gran morbimortalidad y gasto sanitario, así como se demuestra en nuestro reporte, en el que la mayor parte de los pacientes fallecieron producto de las complicaciones propias de la infección y un porcentaje menor secundario a la cirugía.

Finalmente, consideramos que se necesitan estudios adicionales sobre este tema, con un mayor número de pacientes para representar de manera adecuada nuestra realidad nacional. 
1. WENZEL R, EDMOND M. The impact of hospital-acquired bloodstream in- fections. Emerg Infect Dis. 2001;7:174-7.

2. TLEYJEH I, ABDEL-LATIF A, RAHBI H, SCOTT C, BAILEY K, STECKELBERG J, et al. A systematic review of population-based studies of infective endocarditis. Chest 2007; 132:1025

3. PANT S, PATEL NJ, DESHMUKH A, GOLWALA H, PATEL $\mathrm{N}$, BADHEKA A, et al. Trends in infective endocarditis incidence, microbiology, and valve replacement in the United States from 2000 to 2011. J Am Coll Cardiol 2015; 65:2070.

4. OYONARTE M, MONTAGNA R, BRAUN S, ROJO P, JARA J, CERECEDA M, et al. Endocarditis infecciosa: características clínicas, complicaciones y mortalidad en 506 pacientes y factores pronósticos de sobrevida a 10 años (1998-2008). Estudio cooperativo nacional en endocarditis infecciosa en Chile (ECNEI-2). Rev Med chile 2012; 140: 1517-1528.

5. SELTON-SUTY C, CÉLARD M, LE MOING V, DOCO-LECOMPTE T, CHIROUZE C, et al. Preeminence of Staphylococcus aureus in infective endocarditis: a 1-year population-based survey. Clin Infect Dis 2012; 54:1230.

6. CASTILlO FJ, ANGUita M, CASTILlO JC, RUIZ M, MESA D, SUAREZ DE LEZO J, et al. Changes in Clinical Profile, Epidemiology and Prognosis of Left-sided Native-valve Infective Endocarditis Without Predisposing Heart Conditions. Rev Esp Cardiol (Engl Ed) 2015; 68:445.

7. HILL E, HERIJGERS P, CLAUS P, VANDERSCHUEREN S, HERREGODS M, et al. Infective endocarditis: changing epidemiology and predictors of 6-month mortality: a prospective cohort study. Eur Heart J 2007; 28:196

8. MATHEW J, ADDAI T, ANAND A, MORROBEL A, MAHESHWARI P, FREELS S, et al. Clinical features, site of involvement, bacteriologic findings, and outcome of infective endocarditis in intravenous drug users. Arch Intern Med 1995; 155:1641.

9. WILSON W, TAUBERT K, GEWITZ M, LOCKHART P, BADDOUR L, LEVISON M, et al. Prevention of infective endocarditis: guidelines from the American Heart Association: a guideline from the American Heart Association Rheumatic Fever, Endocarditis, and Kawasaki Disease Committee, Council on Cardiovascular Disease in the Young, and the Council on Clinical Cardiology, Council on Cardiovascular
Surgery and Anesthesia, and the Quality of Care and Outcomes Research Interdisciplinary Working Group. Circulation 2007; 116:1736

10. DESIMONE DC, DESIMONE CV, TLEYJEH I, CORREA DE SA D, ANAVEKAR D, LAHR B, et al. Association of Mitral Valve Prolapse With Infective Endocarditis Due to Viridans Group Streptococci. Clin Infect Dis 2015; 61:623.

11. FERNÁNDEZ-HIDALGO N, ALMIRANTE B, TORNOS P, PRIGAU C, SAMBOLA A, IGUAL A, et al. Contemporary epidemiology and prognosis of health care-associated infective endocarditis. Clin Infect Dis 2008; 47:1287.

12. DURACK D, LUKES A, BRIGHT D. New criteria for diagnosis of infective endocarditis: utilization of specific echocardiographic findings. Duke Endocarditis Service. Am J Med. 1994 Mar;96:200-9.

13. LALANI T, CHU V, PARK L, CECCHI E, COREY G, DURANTE-MANGONI E, et al. In-hospital and 1-year mortality in patients undergoing early surgery for prosthetic valve endocarditis. JAMA Intern Med. 2013;173:1495-504.

14. CHU V, SEXTON D, CABELL C, RELLER L, PAPPAS P, SINGH R, et al. Repeat infective endocarditis: differentiating relapse from reinfection. Clin Infect Dis. 2005;41:406-9.

15. BAUMGARTNER H, BONHOEFFER P, DE GROOT N, DE HAAN F, DEANFIELD J, GALIE N, ET AL. ESC Guidelines for the management of grown-up congenital heart disease (new ersión 2010). Eur Heart J. 2010;31:2915-57.

16. MURDOCH D, COREY G, HOEN B, MIRÓ J, FOWLER V, BAYER A, et al. Clinical Presentation, Etiology and Outcome of Infective Endocarditis in the 21 st. Century. The International Collaboration on Endocarditis-Prospective Cohort Study. Arch Intern Med 2009; 169: 463-73.

17. MARTÍNEZ-SELLÉS M, MUÑOZ P, ESTEVEZ A, DEL CASTILLO R, GARCÍA-FERNÁNDEZ MA, RODRÍGUEZ-CRÉIXEMS M, et al. Long-term outcome of infective endocarditis in non-intravenous drug users. Mayo Clin Proc. 2008;83:1213-7.

18. GÁLVEZ-ACEBAL J, RODRÍGUEZ-BAÑO J, MARTÍNEZ-MARCOS F, REGUERA J, PLATA A, RUIZ J, et al. Prognostic factors in left-sided endocarditis: results from the Andalusian multicenter cohort. BMC Infect Dis. 2010;10:17 
19. HOEN B, ALLA F, SELTON-SUTY C, BÉGUINOT I, BOUVET A, BRIANPON S, et al. Associaton pour l'Etude et la prevention de l'Endocardite infectieuse (AEPEI) study group. Changing the profile of infective endocarditis: results of a 1-year survey in France. JAMA 2002; 288:75-81.

20. BRAUN S, ESCALONA A, CHAMORRO G, CORBALÁN R, PÉREZ C, LABARCA J, et al. Endocarditis infecciosa: análisis de 261 casos y resultados del tratamiento con un enfoque multidisciplinario. Rev Med Chile 2000, v.128 n.7.

21. STOCKINS B, NEIRA V, PAREDES A, CASTILlO C, TRONCOSO A. Perfil clínico-epidemiológico de pacientes con endocarditis infecciosa, período 2003-2010 en el hospital de Temuco, Chile. Rev Med Chile 2012; 140: 1304-1311. 